\title{
Means and ends of control
}

\section{Lind, Morten}

\section{Published in:}

IEEE Conf. Systems Man and Cybernetics

Link to article, DOI:

10.1109/ICSMC.2004.1398406

Publication date:

2004

\section{Document Version}

Publisher's PDF, also known as Version of record

Link back to DTU Orbit

\section{Citation (APA):}

Lind, M. (2004). Means and ends of control. In IEEE Conf. Systems Man and Cybernetics (Vol. 1). IEEE. https://doi.org/10.1109/ICSMC.2004.1398406

\section{General rights}

Copyright and moral rights for the publications made accessible in the public portal are retained by the authors and/or other copyright owners and it is a condition of accessing publications that users recognise and abide by the legal requirements associated with these rights.

- Users may download and print one copy of any publication from the public portal for the purpose of private study or research.

- You may not further distribute the material or use it for any profit-making activity or commercial gain

- You may freely distribute the URL identifying the publication in the public portal

If you believe that this document breaches copyright please contact us providing details, and we will remove access to the work immediately and investigate your claim. 


\title{
Means and Ends of Control ${ }^{*}$
}

\author{
Morten Lind \\ $\emptyset$ rsted•DTU, Automation \\ Technical University of Denmark \\ DK-2800, Kongens Lyngby \\ mii@oersted.dtu.dk
}

\begin{abstract}
Means-end analysis plays a significant role in cognitive engineering and has been successfully applied in several work domains, usually in the form of Rasmussens abstraction hierarchy. However, work domains with embedded controls create problems and modifications of the abstraction hierarchy has been proposed to circumvent the problems. But embedded controls is a deep problem for meansend analysis in general and not only for instances like the abstraction hierarchy. It is pointed out that means-end analysis is currently not used in control engineering but should be used to clarify modelling assumptions. Means-end analysis also lacks a proper definition of the control concept. It is proposed that control is defined as a binary relation that assign functional roles to subsystems. This control concept leads to distinct but entangled process-and control hierarchies. It is argued that the problems of embedded control can be resolved by an analysis of the relations between the two types of hierarchy. Illustrative modelling examples are included.
\end{abstract}

Keywords: Means-end analysis, cognitive engineering, human supervisory control, automation design, hierarchies

\section{Introduction}

When designing the human machine interface for the process operator the aim is to represent the process and the automated controls in a manner that suits the needs of the operator in his task. Since the operator is supposed to operate the plant, make decisions and act appropriately in critical situations it is necessary for him to know the means and ends of plant operation i.e. goals and functions for systems and subsystems and the means available for intervention and control i.e. to understand the system as an object of action and to recognize the intention of other actors (automated controls or other operators) in the system. A human supervisor is also dependent on knowledge about the dynamical properties of the system but only to the extent and to a level which is relevant for his task. Methods for cognitive engineering of human machine interfaces propose means-end analysis as a basis for design of the interface to the human supervisor [17][21].

${ }^{*}$ 0-7803-8566-7/04/\$20.00 (c) 2004 IEEE.
Many researchers have applied means end analysis with success, some of them in the form of the abstraction hierarchy [17]. However, some researchers (e.g. [14]) have reported about problems using the abstraction hierarchy for work domains with embedded physical or human control agents. The problems may be circumvented by various adaptations or extensions of the abstraction hierarchy. However, the problem with embedded controls are deeper and not only connected with particular deficiencies of the abstraction hierarchy. It is also a problem for means-end analysis in general [10].

\subsection{The problems of embedded control}

The problem with means end analysis of work domains with embedded control systems does not have a simple solution. It requires consideration of several connected issues. Four issues are discussed below which we believe to be of major importance. The first two issues are primary and concerns the relation between concepts of control and concepts of means-end analysis. A definition of the control concept is suggested which is coherent with the basic ideas of means end analysis and the two following issues elaborate some consequences of this definition. The problem of embedded controls with the abstraction hierarchy originated in work domain analysis of intensive care units. The relevance of the issues to means end analysis of physiological systems will therefore be indicated where appropriate.

\subsubsection{Current control engineering concepts}

Current control engineering concepts does not match particularly well with concepts of means end analysis. It is accordingly difficult to translate the design rationale for a control system into the vocabulary of means, ends, purposes and functions. As a consequence, it becomes questionable how to use the why, what and how distinctions of meansend analysis to make the control system intelligible for the operator. In addition, control system objectives are often indirectly related to the real objectives or goals due to limitations of the plant instrumentation. The translation problem is mainly caused by a general lack of insight in the cognitive aspects of process modelling and problem framing in the control engineering community. The problem is of particular importance for cognitive engineering because the designer 
must reverse engineer descriptions of goals and functions of the automated controls and the process from available informal and often incomplete plant documentation. However, as discussed later in the paper, it is also a problem for control engineering that needs to be addressed.

This problem is of particular difficulty in physiological systems since there is no blueprint or designer to specify the purposes and functions of the physiological system and its parts. The analyst must therefore induce this information from the available but often incomplete knowledge of the structure, behavior and evolution of the physiological system.

The translation problem will be approached below by using Goffmann's [1] frameworks of interpretation to analyze the background knowledge required for building differential equations in control engineering.

\subsubsection{The control concept and means-end analysis}

It is also a problem that the concept of control has many interpretations and that its particular meaning in means-end analysis is not well defined. We suggest that control is defined as a directed relation from a causal agent (the controlling subsystem) to an object (the process subsystem under control). The control relation implies an assignment of functional roles to two systems, one serving the role of being the process under control and the other the role of being the controller. The distinction between the two functional roles is important because each role defines a view or particular end-context of the means-end analysis. The two views will be called the process view and the control view in the following. The process view provides an analysis of purposes, functions and structure of the process and the control view account for the purposes, function and structure of the control systems. The two views lead accordingly to two distinct hierarchies. It should be noticed that the distinction between the two hierarchies is purely functional i.e. it does not represent a structural decomposition of the system. This means that the control system will be represented within the process view as a means for achieving process ends. Conversely, a process will be represented in the control view as a means for intervention and observation.

\subsubsection{Entangled hierarchies}

The control and the process hierarchies are entangled in several ways of which we will consider only three here; 1) a control system can have a constitutive role in the formation of a functional level in the process hierarchy, 2)it can serve as a means of transfer between two different hierarchical organizations of the process functions and 3) process components and subsystems may serve both process and control functions. Other more complex relations between the hierarchies are discussed in [5]. The distinction between the two types of hierarchy and their entanglement will be discussed below by a process example with an embedded control system (the so-called Mariotte bottle).

\subsubsection{Assignment of roles to subsystems}

It is not always obvious how to assign the object and agent roles of the control relation to subsystems when modelling means-end relations in a system. In many cases, the technology applied will indicate the role of a particular subsystem but in other cases it is not at all obvious. For example, electronic circuits in a process plant will without doubt serve control functions whereas the role of e.g. hydraulic systems is less certain because it can have both roles. Sometimes systems serve both as control agents and as objects of control at the same time. The problem of role assignment will also be exemplified by the Mariotte bottle.

The assignment of functional roles is a serious problem in physiological systems as it can be difficult to distinguish the processes from the controls in such systems. Especially control functions which are realized by enzymatic and hormonal reactions are difficult to distinguish from the metabolic processes.

\section{The translation problem}

In current approaches to control engineering, process modelling is seen as the use of mathematical equations to represent the physical properties and constraints defining the dynamics of the process under control. The mathematical equations are used to develop the algorithms to be implemented into control software and hardware. Researchers in control engineering have provided new insights in process modelling that goes beyond the equations (see e.g. [2]) and there is also a recognition of the necessity to know purposes and functions of the process under control. This knowledge is not subject of explicit formalization but nevertheless it is considered relevant for the modelling problem because it defines assumptions and the aspects of the system which are relevant for the control problem and therefore to include in a dynamic model of the process. There is therefore a need for using means-end analysis in process modelling in order to address these background assumptions explicitly.

Presently the state of art in formalized means-end analysis is not as advanced as the tools available for analysis of systems dynamics and Multilevel Flow Modelling $[6,8]$ is one of the few methodologies available for means-end analysis of control systems. However, the situation is slowly changing due to the influence of artificial intelligence in control [7] and to an increased academic (see e.g. [12]) and industrial interest in design of plant level control solutions [3,4]. Furthermore, the design of plant wide control systems is much more dependent on a formalized analysis of system purposes and functions than low level feedback controls because they are directly related to the overall goals of plant operation and less dependent on physical constraints.

\subsection{Frameworks of interpretation}

The role of means end concepts in modelling process dynamics can be revealed by realizing that the modelling activity in addition to the mathematical aspects also involve a cognitive process of interpretation. By the interpretation 
the modeler make sense of the events and phenomena in the problem under investigation. However, according to Goffman [1] we can distinguish between two primary frameworks of interpretation. A framework of interpretation serve as a frame of reference and is seen as rendering what would otherwise be a meaningless and chaotic situation into something that is meaningful and with structure. The two primary frameworks are called the natural and the social frameworks and are defined as follows:

...Natural frameworks identify occurrences seen as undirected, un-oriented, unanimated, unguided "purely physical". Such unguided events are ones understood to be due totally, from start to finish, to "natural" determinants. It is seen that no willful agency causally and intentionally interferes, that no actor continuously guides the outcome. Success or failure in regard to these events are not imaginable...

...Social frameworks, on the other hand, provide background understanding for events that incorporate the will, aim, and controlling effort of an intelligence, a live agency, the chief one being the human being...What the agent...does can be described as "guided doings". These doings subject the doer to "standards," to social appraisal of his action based on its...efficiency, economy, safety..., and so forth.

Searle [19] has proposed another related distinction between brute and social facts and argues that descriptions of function and purposes of physical equipment are social facts because they refer to human goals and intentions. Searle also point out that descriptions of the function of a physical device must ultimately refer to its physical properties i.e. to so-called brute facts. Descriptions given within Goffmann's two frameworks may therefore use the same terms but will have different meanings depending on whether they refer to objective observable physical events or states (brute facts) or to inter-subjective intentions (social facts). A shown later, this insight is crucial in order to understand how the two frameworks of interpretation interplay when modelling the dynamics and functional organization of a physical artifact.

\subsubsection{Remarks concerning the abstraction hierarchy}

Goffmann's frameworks has, on a superficial level, connections with some of the key ideas of Rasmussen's abstraction hierarchy. The reader might therefore wonder why Goffmann's work is used here rather than the abstraction hierarchy which is well known in the cognitive engineering community. There are several reasons for this decision. Firstly, it is necessary to distinguish between means end analysis as a generic type of analysis and the abstraction hierarchy as an instantiation hereof with particular strengths and weaknesses. Secondly, Goffmann's social framework of interpretation is entirely consistent with the general concepts of means end analysis. Thirdly, Goffmann's clear distinction between the two frameworks makes it possible to analyze how control problems are framed and how levels of function emerge through an interplay between the two corresponding modes of interpretation. This type of analysis is not possible in the abstraction hierarchy because levels here appear to be predefined or not problematic. Finally, the abstraction hierarchy merge the process and the control hierarchies (not unlike two deck of cards) by locating control functions on the level of generalized function together with process functions like cooling. The abstraction hierarchy therefore eliminate the distinction between types of hierarchy we want to emphasize. By using Goffmann's frameworks of interpretation we are accordingly able to see the problem with means end analysis of control systems more clearly.

\subsection{How control problems are framed}

Goffmann explains with this distinction why natural frameworks of interpretations (such as physics and chemistry) are not sufficient for understanding control systems. We can describe automated control systems as purely physical since they ultimately rely on hardware, but we need the social framework to characterize the goal directed nature of control actions i.e. to the capture the intentionality. We can accordingly apply the two primary frameworks to provide different descriptions of a control system. These descriptions have distinct meanings by referring to different frameworks of interpretation.

The physical framework can be used to explain how the control is working i.e. as an un-oriented and unguided activity. This is the framework of interpretation advocated by control engineers and theorists referring to the natural sciences as a prerequisite for the analysis and synthesis of dynamic systems. However, the social framework is required if we want to describe the control as an activity directed towards the achievement of purposes and goals - a view advocated by researchers in cognitive science [16] and artificial intelligence interested in intentional systems [22] and the view underlying means-end analysis. The importance of the social framework for interpretation control systems is also stressed by Rescher [18] when he argue that the concept of control is inherently connected with concepts of intention, purpose and goal. But, as demonstrated below we need also to know how the process is operated and the purposes and functions served by its subsystems and equipment. Both frameworks are accordingly relevant for understanding both the process and the control and the social framework is of particular importance in understanding processes with embedded controls. Note that the two primary frameworks of interpretation are not exclusive but complementary. They define different perspectives on the same situation or system which are of relevance in modelling tasks.

\subsection{A simple tank example}

We will in the following illustrate how the two frameworks are used in modelling the dynamics of the simple system 
shown in Figure 1. The use of the frameworks cannot be illustrated in a direct way because all attempts to do so invariably will imply implicit selections of features of the situation at hand and be influenced by the purpose of analysis. We therefore explain the ideas by first presenting the result of the modelling i.e. the differential equation that provides a mathematical representation of the tank dynamics. Hereafter, we will reveal how the problem has been framed.

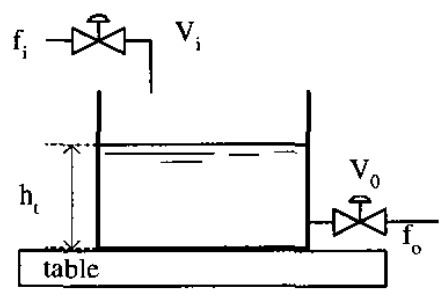

Figure 1: A simple tank example

Let us consider the apparently trivial problem of modelling the dynamics of the water level $h_{t}$ in the tank and its dependencies of the flows of water $f_{i}$ in the inlet valve $V_{i}$ and $f_{o}$ in the outlet valve $V_{o}$. We get the following differential equation:

$$
\frac{d h_{t}}{d t}=\frac{f_{i}-f_{o}}{A \rho}
$$

where $A$ is the cross sectional area of the tank and $\rho$ is the specific mass of the water. Since the equation describe the dynamics of the system in terms of the physical properties of its parts it may seem as if the modelling only involve the application of the natural framework of interpretation. However, the social framework is at play in the background. This can be revealed by analyzing the assumptions made as a prerequisite for setting up the equation.

\subsubsection{The assumptions}

It is assumed that the purpose of the tank is to receive, store (i.e. to contain for a specified period of time) and deliver water. It is also assumed that the valves and the associated piping serve these purposes by transporting the water so that the tank can receive and deliver the water. The differential equation describe the ability of the tank to receive (by $f_{i}$ ), contain (by $h_{t}$ and $A$ ) and deliver (by $f_{o}$ ) the water. We have also assumed that the water is a moveable and storable substance and that it is fluid so that it can adapt its spatial form to the tank volume (by $\rho$ ). We have also assumed the existence of an agent (an operator or an automated system) which can determine or control the flows of water $f_{i}$ and $f_{o}$ and therefore, by the causal influence on $\frac{d h_{t}}{d t}$ expressed in the equation, exercise control on the rate of change of the level $h_{t}$. Finally we have also assumed that the system is placed in a field of gravity otherwise the water would not be received by the tank when it leave the inlet valve. In addition, water present in the tank would not be able leave it because there would be no hydrostatic pressure to force it to flow through the outlet valve. Notice that the assumptions mentioned above comprises both brute and social facts.

It is realized that even though equation (1) represents relations between physical properties its formulation require knowledge of the functions served by system parts in the fulfillment of the purpose of the whole. But this knowledge about the means and the ends of the system is not explicitly represented in the equation and only described informally above in natural language. We propose that this implicit means-end knowledge should be made explicit in a formalized form in order to make it clear how the process is conceived as an object of control (i.e. within a social framework) and thereby make the control semantics of the system transparent.

The implicit assumptions seems to be trivial but they are not, even for the simple system we are considering. Purposes and thereby the functions of system parts are often not clearly described or even identified. The system may even be used simultaneously or in different situations for (possibly conflicting) different ends. For example, the inlet water could be used to heat the tank. In such a situation we should also consider the thermodynamic properties of the tank and the water in order to account for the dynamics. Furthermore, in the example there is a direct relation between the purpose of the system and the properties and the dynamics expressed in (1) but in other situations the relation could be more indirect. For example, if the purpose of the valves and the tank in Figure 1 is to prevent the water from falling on the table i.e. to be a barrier against loss of water, the equation would only describe a way or manner of preventing this situation and not relate directly to the purpose itself. In such situations an explicit representation of the purpose would clearly be required to make the purpose or meaning of the system transparent. A logic analysis of these description problems is presented in $[9,11]$.

It is realized that the implicit relations between the qualitative means-end analyzes and the quantitative mathematical models of plant dynamics (if available) make it difficult to use the control engineering information about plant dynamics in way that is consistent with the principles of cognitive engineering.

\subsection{Representing means and ends}

Natural language was used above to describe the purpose of the tank system and the function of its various parts. Actually, natural language is very effective at describing meansend and functional relations. A variety of means-end relations can be expressed through so-called semantic roles (object, agent, instrument, counteragent, ...)connected with action verbs [13]. Natural language allow us also to assign different semantic roles to objects and actors in a situation depending on the perspective under which it is seen. Thus, if the purpose in the example above is instead to heat the tank walls by the water, the semantic roles would be different. The tank would be the object of the heating action and the water would have the role of being an instrument or a 
means of heat transfer. The purpose in view determine the roles of the tank and the water and is expressed explicitly in the semantic structure.

Modelling concepts and a number of knowledge representation techniques such as frames [15] or conceptual graphs [20] have been developed within artificial intelligence research for representing the semantics of actions. Another possibility is to use Multilevel Flow Modelling [6, 8] which is particularly aimed at modelling process control systems with complex means-end structures. These techniques can be used to model means-end relations and thereby to make explicit models of the conceptualizations that comprise the background assumptions for the formulation of differential equations of a controlled object.

\section{The problem of embedded controls}

The tank example above demonstrated that modelling of the dynamics of physical system imply a control relation (between the tank and its associated equipment and an external control agent). The modelling relied on an understanding of means-end relations within a process view. These insights becomes even more relevant in the following more complex example shown in Figure 2 which have an embedded control system. It will be clear that in such a case, which are in abundance in industry, we need also to apply the control view and to understand how it is related to and entangled with the process view.

\subsection{Mariotte's bottle}

The example is a slightly modified Mariotte's bottle which is used in e.g. chemical laboratories to provide a constant and controllable fluid flow (e.g. for dosing purposes). Human machine interface problems are rarely discussed in connection with such simple devices. It has been chosen here as an example because it illustrates in a direct and simple way the problems with means-end analysis of processes with embedded control systems. The insights obtained from the example are believed also to be valid for more complex systems.

The overall purpose of the Mariotte's bottle is to deliver water at a constant but controllable flow rate. The system comprises a closed tank (instead of a bottle) containing the water, an inlet valve $V_{i}$, an outlet valve $V_{o}$, a vent valve $V_{v}$ and a pipe which provides an open path from the tank interior to an environment with constant pressure $P_{e}$. The valves are assumed to be of the on-off type. The inlet valve is used for filling the tank and the depth of immersion of the pipe $d_{b}$ determines the outlet pressure $p_{o}$ and hereby the outlet flow $f_{o}$ (assuming that the outlet valve is open). Note that the inlet and the vent valves are not present in the original device. They have been added here in order to facilitate the discussion of filling and emptying operations. In spite of the modifications we will for convenience refer to the system as Mariotte's bottle in the following.

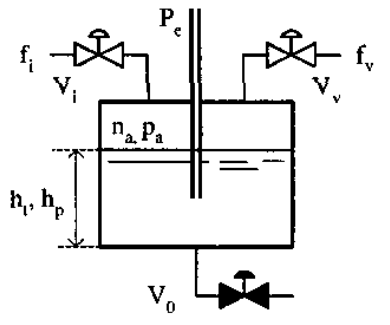

(A) Fill tank

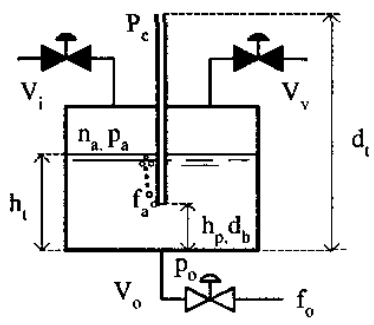

(C) Deliver water

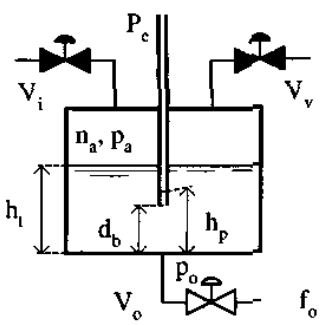

(B) Enable regulation

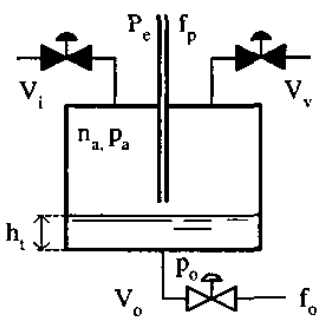

(D) Empty tank
Figure 2: Mariottes bottle and its four configurations (open valves are white and closed are black)

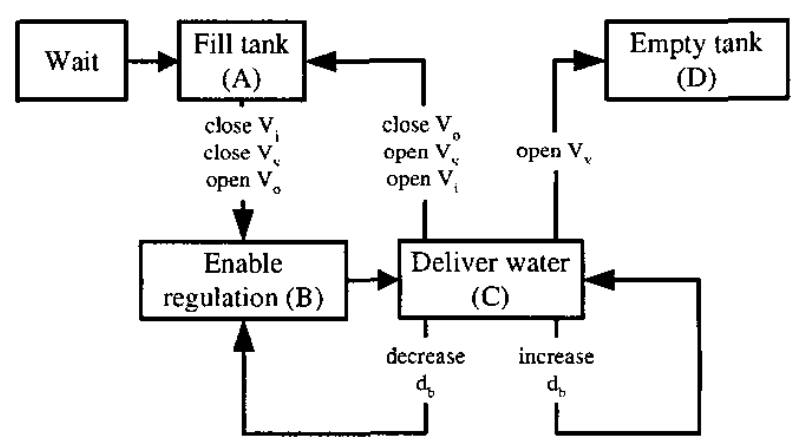

Figure 3: Phases of operation of Mariotte's bottle

\subsection{Phases of operation}

The system is operated as a batch system with the four phases shown in Figure 3. The operator can change the operating phase of the system by control actions (closing and opening the valves and by shifting the position of the pipe). The configurations of the system in the four phases are shown in Figure 2. Note that the operating objective and thereby also the assignment of the functional roles of the system also are dependent on the phase. The phases and the associated functions of the subsystems are described below in detail.

\subsubsection{Fill tank (A)}

The objective in phase $\mathrm{A}$ is to fill the tank with water. This is done by opening the inlet valve $V_{i}$ for a period of time and then closing it. The outlet valve $V_{o}$ is kept closed but 
the vent valve $V_{v}$ is open until the filling operation has been completed so that the air can escape from the tank when it is displaced by the water. The function of the tank in this phase is simply to store the water. The inlet valve $V_{i}$ has the function of transporting the water into the tank. It serves also as a means for control (actuator). The vent valve $V_{v}$ has the function of transporting the air.

\subsubsection{Enable regulation (B)}

The objective in phase $\mathrm{B}$ is to establish working conditions for a regulation mechanism which keep the outlet pressure $p_{o}$ (and thereby the flow $f_{o}$ ) at a constant value (see phase $\mathrm{C}$ below). The pipe is first immersed into the water to a depth $d_{b}$ corresponding to the desired pressure. By opening the outlet valve $V_{o}$, the water level $h_{t}$ is reduced until it reaches the lower end of the pipe $d_{b}$ and air can enter the bottle (the working condition for the regulation). The pressure of the enclosed air $p_{a}$ in the bottle will at the same time be reduced from the initial environmental pressure $P_{e}$ to a value that, together with the hydrostatic pressure produced by the water, provides the desired $p_{o}$. This phase is entered when the inlet and vent valves are closed and the outlet valve is opened after the filling phase, or when the set-point $d_{b}$ for the outlet pressure is decreased (see below). The tank has two (interdependent) functions in this phase - to store water and air. Note that whereas the pipe had no function in phase $A$ it has two functions in this phase. It has the (process) function of transporting water and the (control) function to indicate tank air pressure. The air pressure can be read by the operator as the distance between the water levels in the tank $h_{t}$ and in the pipe $h_{p}$. The functions of the outlet valve $V_{i}$ in this phase are to transport the water and to be a means for control (actuator).

\subsubsection{Deliver water $(C)$}

The objective of phase $C$ is to deliver water under a constant outlet pressure. The pressure is regulated (e.g. maintained) by a bubble mechanism which compensate for the decrease in pressure caused by the outflow of water by letting in air from the pipe so that the air pressure $p_{a}$ in the bottle increased. The set-point for the outlet pressure is determined by the distance $d_{b}$ from the tank bottom to the lower end of the pipe. Note that in the other phases, the outlet pressure is dependent on the level of water in the tank $h_{t}$ and independent of the pipe position. The functions of the tank and the outlet valve are the same as in phase B. Note however, that the pipe now serves two other functions. Its position $d_{b}$ indicates the set point for the outlet pressure $p_{o}$ (control function) and it provides a transport function (process) for the ingoing air.

\subsubsection{Empty tank (D)}

In phase D the objective is to reduce the water level $h_{t}$ to zero. This is simply done by letting the water leave the tank through the outlet valve. The functions of the tank and the outlet valve are the same as in phase B. Note however, that the pipe now serves only one (process) function namely to transport air into the tank.

\subsection{Control actions}

The transition between the phases of the Mariotte bottle is determined by control actions as indicated in Figure 3 . We will only discuss two of the actions (decrease $d_{b}$ and increase $d_{b}$ ) which are of particular interest for the analysis below of the problem with embedded controls.

When the operator decreases the set-point for the outlet pressure $p_{o}$ by decreasing $d_{b}$, the water will rise in the pipe and prevent the air bubbles to pass. The regulation mechanism is accordingly deactivated. However, the regulation becomes active again after a while without further intervention by the operator because the water level $h_{t}$ decreases until it reaches the new value of $d_{b}$ due to the outflow of water. The operator can therefore let the system reestablish the regulation by its own dynamics. This means that the new output pressure level is determined by $d_{b}$ and they are therefore (after a time delay) connected by a causal relation.

When the operator increases the pressure set-point by increasing $d_{b}$ the regulation mechanism remains active so that the outlet pressure change in direct dependence of the pipe position (assuming that the operator is not moving the pipe too fast). There will accordingly be a direct causal relation between an increase in $d_{b}$ and an increase in the outlet pressure $p_{o}$.

\subsection{The means-end analysis}

The descriptions above of the phases and its associated objectives and subsystem functions contain the information which is needed in order to identify means-end relations and levels of function in the Mariotte bottle. Figure 4 depict the result of an analysis of the means-end relations adapting the symbology of Multilevel Flow Modelling [8]. It should be noted that the model is semi formal and tentative and only suited to illustrate the problems with embedied control systems and not meant to provide the solution. Further work is required in order to resolve all aspects of the problem. We have also excluded parts of the model that are not significant for the issues addressed. We have accordingly only included the objectives and functions related to phases $\mathrm{B}$ (enable regulation) and $\mathrm{C}$ (deliver water) and the operator control actions connecting the two phases (decrease $d_{b}$ and increase $d_{b}$ ).

The resulting model in Figure 4 is best explained by starting with the sub-model representing phase $\mathrm{B}$ (right hand bottom corner). This sub-model comprises the process functions involved in the transport and storage of water and air, a control function and an objective $q$ which is achieved as the joint interaction of the two functions. The control objective is to produce the end-condition $h_{p}<d_{b}$ i.e. to lower the water level in the pipe until the air can enter into the bottle. This end-condition enters as a pre-condition for two other functions described below.

Continuing the explanation with phase $\mathrm{C}$ it is seen that the end-condition $q$ for phase $\mathrm{B}$ is a pre-condition for a control 


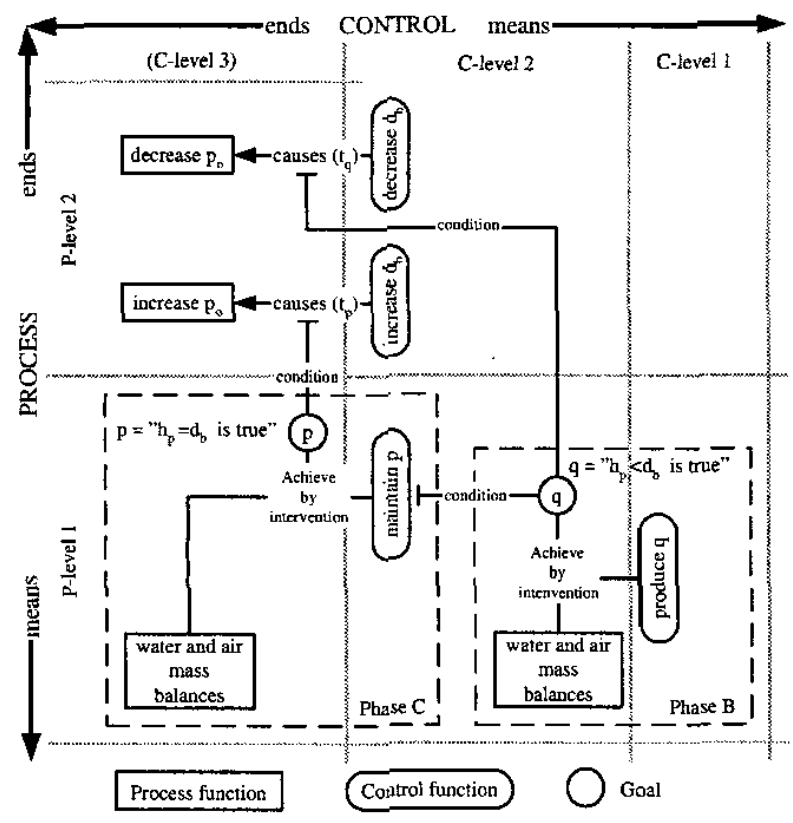

Figure 4: Process and control hierarchies (phases B and C)

function. This control function represents the function of the regulation mechanism which is to maintain $p$, the condition that ensure that outlet pressure is held at a constant value corresponding to $d_{b}$. The process function comprises as above the subsystem functions involved in transport and storage of water and air. However, note that the configuration of the system in the two phases are slightly different (see descriptions above of the two phases).

Moving to the top left corner of the model we can see that it contains two sub-models with similar structures. They both comprises a process function related to a control function by a causal relation. The control functions represent two ways of moving the pipe and the causally related process functions represents the corresponding response of the process i.e. the decrease or increase of the outlet pressures. It is also seen that the two causal relations are conditioned by endconditions $p$ and $q$. This means that the causal relation is only existing provided the corresponding condition is satisfied. This sub-model describe accordingly the total system as a control device namely an actuator for the outlet pressure.

\subsubsection{Hierarchies and functional levels}

It is clearly seen that the model comprises two entangled functional hierarchies that each require the adoption of process and control views. In the vertical direction we have two levels of process function (P-level 1 and P-level 2). Along the horizontal direction we can identify two levels of control (C-level 1 and C-level 2). Note that the number of levels are not always the same (a control function could provide a condition for another control function etc.)

We have also indicated an optional control level which should be introduced if the operation of Mariotte's bottle was changed so that outlet flow (pressure) was controlled to match the inflow, assuming e.g. a continuous filling with fluctuations in the inflow (so-called cascade control). This would also introduce a new level of process function because the total system now would be behave as a transport system!

\section{Conclusions}

The paper presents an analysis of problems with meansend analysis of dynamic work domains with embedded controls. It is pointed out that means-end analysis is currently not used in control engineering but should be used to clarify assumptions made in building differential equation models for control design. Better communication of design intentions could then be obtained with cognitive engineering professionals. The paper demonstrates by examples the reasons why embedded control systems create problems with means-end analysis. It is shown that it is necessary to distinguish clearly between process hierarchies and control hierarchies and that these two hierarchies are entangled in nontrivial ways. It is also demonstrated that embedded controls has a central role in the formation of functional levels in the process hierarchy.

\section{References}

[1] E. Goffman, Frame Analysis, Penguin Books, London, 1974.

[2] K. Hangos and 1. Cameron, Process Modelling and Model Analysis, Academic Press, San Diego USA, 2001.

[3] ISA, "Batch control part1: Models and terminology", ANSI//SA-88.01.1995, Instrument Society of America, 1995.

[4] ISA, "Enterprise-control system integration part1: Models and terminology", ANSI/ISA-95.00.01-2000, Instrument Society of America, 2000.

[5] M. Lind, "Functional architectures for systems management and control", In M. Lind, editor, Interactive Planning for Integrated Supervision and Control in Complex Plant. Final report - Project 4937-92-08-ED ISP DK. Institute for Systems Engineering and Informatics, CEC Joint Research Centre, Ispra Italy, 1993.

[6] M. Lind, "Modeling goals and functions of complex industrial plant", Applied Artificial Intelligence, Vol 8, No. 2, pp. 259-283. 1994.

[7] M. Lind, "Status and challenges of intelligent plant control", Annual Review of Control, Vol 20, pp. 23-41. 1996.

[8] M. Lind, "Plant modeling for human supervisory control", Trans. of the Institute of Measurement and Control, Vol 21, No. 4-5, pp. 171-180. 1999. 
[9] M. Lind. Promoting and opposing. NKS-R-07 project report, Ørsted DTU, Technical University of Denmark, 2002.

[10] M. Lind, "Making sense of the abstraction hierarchy in the power plant domain", Cognition Technology and Work, Vol 5, No. 2, pp. 67-81, 2003.

[11] M. Lind. Description of composite actions - towards a formalization of safety functions. NKS-R-07 project report, Ørsted DTU, Technical University of Denmark, 2004.

[12] W. L. Luyben, B. J. Tyréus, and M. L. Luyben, Plantwide Process Control, McGraw Hill, New York USA, 1999.

[13] J. Lyons, Semantics: 2, Cambridge University Press, Cambridge USA, 1977.

[14] A. Miller and P. Sanderson, "Modeling "deranged" physiological systems for ICU information system design", Proc. HFES/IEA 2000 Human Factors and Ergonomics Society, San Diego USA, July-August. 2000.

[15] M. Minsky, The Society of Mind, Simon and Schuster, New York USA, 1985.

[16] A. Newell and H. Simon, Human Problem Solving, Prentice Hall, New Jersey USA, 1972.

[17] J. Rasmussen, A. M. Pejtersen, and L. P. Goodstein, Cognitive Systems Engineering, John Wiley New York, 1994.

[18] N. Rescher, Essays in Philosophical Analysis, University of Pittsburgh Press, Pittsburgh USA, 1969.

[19] J. R. Searle, The Construction of Social Reality, The Free Press, New York. 1995.

[20] J. F. Sowa, Knowledge Representation; Logical, Philosophical and Computational Foundations, Brooks/Cole, Pacific Grove USA, 2000.

[21] K. J. Vicente and J. Rasmussen, "Ecological interface design: Theoretical foundations", IEEE Transactions in Systems Man, and Cybernetics, Vol 22, No. 4, pp. 589-606, 1992.

[22] M. Wooldridge, Reasoning About Rational Agents, The MIT Press, Cambridge, Massachussets USA, 2000. 